\title{
Innate Immune Responses in the Neutrophils of Community Dwelling and Nursing Home Elders
}

Manisha Juthani-Mehta ${ }^{1 *}$, Xiuyang Guo $^{2}$, Albert C. Shaw $^{1}$, Virginia Towle ${ }^{1}$, Yuming Ning ${ }^{1}$, Xiaomei Wang $^{1}$, Heather G. Allore $^{1}$, Erol Fikrig ${ }^{1}$ and Ruth R. Montgomery ${ }^{1}$

${ }^{1}$ Yale University School of Medicine, USA

${ }^{2}$ Roger Williams Medical Center, Boston University School of Medicine, USA

\begin{abstract}
Objective: To evaluate innate immune responses of older disabled nursing home residents that may contribute to infectious disease susceptibility, we compared surface markers and signaling efficiency of neutrophils from nursing home residents and community dwelling elders.

Design: Observational pilot study.

Setting: Five New Haven, CT area nursing homes and the greater New Haven community.

Participants: 15 nursing home residents and 43 community dwelling elders.

Measurements: Neutrophils were isolated and Toll-like receptor (TLR) and $\beta 2$ integrin expression on the surface of unstimulated neutrophils were measured via flow cytometry. Chemokine induction was determined by Quantitative PCR.

Results: Surface expression of TLR4 was elevated among nursing home residents compared to community dwellers (mean percent positive cells 33.91 [SE 2.75] vs. 15.67 [SE 1.58], $p<0.001$ ), while expression of the $\beta 2$ integrins CD11b and CD18 was significantly lower (mean fluorescent intensity 460.8 [SE 49.1] vs. 632.9 [SE 29.5] for CD11b and 59.6 [SE 7.9] vs. 137.6 [SE 4.6] for CD18, $p<0.0001$ ). Neutrophils from nursing home residents produced substantially reduced levels of chemokines at baseline and after stimulation.

Conclusions: Because integrins are an important pathway to phagocyte signaling and contribute to adherence and locomotion of neutrophils, reduced $\beta 2$ integrin expression may contribute to impaired responses to stimulation and reduced adhesive properties in PMN from nursing home residents. Since integrin CD11b has been shown to negatively regulate TLR4 response, it is plausible that lower levels of CD11b contribute to elevated expression of TLR4.
\end{abstract}

Keywords: Innate immunity; Nursing homes; Community dwellers

\section{Introduction}

Nursing home residents represent a frail and disabled population that is susceptible to many infectious diseases, most commonly pneumonia and urinary tract infection (UTI) [1]. Immunosenescence, in part, places these residents at increased and greatest risk for bacterial infections [2]. The innate immune system is the first line of host defense against infectious agents. Polymorphonuclear leukocytes (PMN, neutrophils), a key component of the innate immune response to infection, are the most abundant type of leukocyte in humans, and are the first immune cells to be recruited to inflammatory foci [3]. They ingest pathogens by phagocytosis, release antimicrobial peptides stored in granules, and produce reactive oxygen species and extracellular traps to combat numerous pathogens [3].

Age-related deficiencies of the innate immune system include reduced recruitment, phagocytosis, granule release, and microbial activity by PMN or macrophages, suggesting an age-related dysfunction in signal transduction as a manifestation of immunosenescence $[4,5]$. We have previously examined expression of Toll-like receptors (TLRs), key to recognition of conserved patterns of bacterial and viral pathogens, and have shown an age-related decrease in expression of certain TLRs in monocytes and dendritic cells, $[6,7]$ elevation in TLR5, [8] and age-related dysregulation of TLR3 in macrophages[9] among healthy community-dwelling elders.

To date, the innate immune responses of nursing home residents have not been explored. Altered responses in these aged, most frail, individuals may contribute to infectious disease susceptibility. In this study, we assessed PMNs from a population of disabled nursing home elders compared to healthy community dwelling elders for surface expression and functional efficiency of TLRs and $\beta 2$ integrins, a family of cell surface receptors that mediate adhesion and are up-regulated during activation of innate immune responses $[10,11]$.

\section{Methods}

\section{Setting and participants}

Nursing home participants were from five New Haven area nursing homes that were participating in a prospective cohort study that has been previously described [12]. A subset of thirty seven participants (or their surrogates) from nursing homes that agreed to participate in

*Corresponding author: Manisha Juthani-Mehta, Associate Professor, Yale University School of Medicine, New Haven, CT 06520, USA, E-mail: manisha.juthani@yale.edu

Received January 16, 2014; Accepted January 21, 2014; Published January 27 2014

Citation: Juthani-Mehta M, Guo X, Shaw AC, Towle V, Ning Y, et al. (2014) Innate Immune Responses in the Neutrophils of Community Dwelling and Nursing Home Elders. Aging Sci 2: 115. doi: 10.4172/2329-8847.1000115

Copyright: () 2014 Juthani-Mehta M, et al. This is an open-access article distributed under the terms of the Creative Commons Attribution License, which permits unrestricted use, distribution, and reproduction in any medium, provided the original author and source are credited. 
this sub-study was approached for consent to obtain a blood sample. Fifteen participants provided written consent. Samples from forty three healthy community dwelling elders on whom neutrophils were available were obtained from a study of the effects of aging on innate immune responses. This study was approved by the Yale University Human Research Protection Program.

\section{Data collection}

At the five nursing home facilities, age, sex, race, and comorbidities were obtained from the medical record. Baseline mental status and functional status were assessed through interviews with nursing staff. These interview questions were adapted from the Minimum Data Set (MDS), a reliable and validated questionnaire that must be completed on all nursing home residents [13]. Baseline data from the forty three healthy community dwelling elders obtained via interview were utilized for comparison. Blood samples were obtained by a trained phlebotomist from all fifty eight participants.

\section{Polymorphonuclear leukocyte (PMN) isolation and stimulation}

Heparinized blood was separated by Ficoll-Hypaque density gradient centrifugation and PMNs were purified from the pellet by Dextran density sedimentation and hypotonic lysis of red blood cells [14]. PMN $\left(10^{6} / 100 \mu \mathrm{l}\right)$ were re-suspended in PBS containing $\mathrm{Ca}^{2+}$, $\mathrm{Mg}^{2+}, 0.1 \% \mathrm{BSA}$, and physiologic glucose $(5.4 \mathrm{mM})$ as described previously [14]. Cells were untreated or treated for $30 \mathrm{~min}$ at $37^{\circ} \mathrm{C}$ with TNF- $\alpha, 20 \mathrm{ng} / \mathrm{ml}$ (R\&D Systems, Minneapolis, MN); LPS, $0.5 \mu \mathrm{g} / \mathrm{ml}$ (Sigma); or Pam3CSK4, $5 \mu \mathrm{g} / \mathrm{mL}$ (Invivogen, San Diego, CA).

\section{PMN flow cytometry}

We assayed surface TLRs (TLR1, 2, and 4) for which robust antibody reagents were available at the time of the study. For analyses of surface TLR and integrin expression in unstimulated samples, PMN were labeled for 30 minutes in PBS-BSA at $4^{\circ} \mathrm{C}$ with specific antibodies conjugated as follows: APC-CD15; Fluorescein isothiocyanate (FITC)anti-CD18; Phycoerthryin (PE)-CD11b (Dako Cytomation, Glostrup, Denmark); TLR1 (PE, clone GD2.F4), TLR2 (FITC, clone TL2.1), PETLR4 (eBioscience). The isotypes used were PE-conjugated mouse IgG1K and FITC-conjugated mouse IgG2a K (BD Bioscience). Cells were fixed in $0.5 \%$ paraformaldehyde and stored at $4^{\circ} \mathrm{C}$ until analysis by fluorescence-activated cell sorter (FACS; FACS Calibur, Becton Dickinson, Becton Drive Franklin Lakes, NJ). A total of 10,000 events per tube were acquired and cell populations expressing CD15 were gated for analysis using FlowJo software (Tree Star, Inc., Ashland, Oregon).

\section{Quantitative PCR (Q-PCR)}

Q-PCR using specific primers and probes was carried out as previously described [9]. Total RNA was extracted from PMN using the RNeasy kit (Qiagen, Valencia, CA). RNA was used to synthesize cDNA by the Affinity Script Multi Temperature cDNA synthesis kit (Strata gene, La Jolla, CA). Taq Man gene expression assays for human $I L-8, C C L 4, C C L 20$ and IFN $\gamma$ genes were purchased from Applied Biosystems (Foster City, CA) and $\beta$-actin was used as a control. Values for each gene were calculated from the accompanying standard curve in each Q-PCR reaction plate. Levels of genes were expressed as a ratio to cellular $\beta$-actin cDNA copies measured by Q-PCR. Each duplicate measurement was divided by the corresponding measurement for actin and then averaged. The ratio of the amount of amplified gene compared with the amount of $\beta$-actin cDNA represents the relative levels in each sample.

\section{Statistical analysis}

Proportions, means or medians, where appropriate, were used to describe the demographic and clinical characteristics of the cohort at enrollment. We used multivariable General Linear Models to estimate the effect of participant group (i.e., nursing home residents or community dwellers) on the percent-positive cells expressing specific TLR proteins, controlling for age, sex, and numbers of comorbidities. Results are presented as least squares means to improve clinical interpretation. Statistical tests were 2 -tailed, and $\mathrm{p}<.05$ considered to indicate statistical significance. Analyses used SAS version 9.1 (SAS Institute, Cary, NC). Significance for Q-PCR was assessed by the MannWhitney test using Graph pad Prism (Graph Pad Software, Inc.) as previously described [7].

\section{Results}

\section{Altered levels of TLRs and $\beta_{2}$ Integrins in PMN of nursing home residents}

Table 1 characterizes the 15 nursing home participants and the 43 community-dwelling elders. To examine the immune status of nursing home residents in comparison to healthy community dwelling elderly subjects, we isolated PMN and quantified surface expression of TLR1, 2, and 4. Expression levels of TLRs 1 and 2 were not significantly different between community dwellers and nursing home residents (mean percent positive cells 16.6 [SE 2.1] vs.13.2 [SE 3.6], $\mathrm{p}=0.43$ and 59.5 [SE 3.3] vs. 65.5 [SE 5.8], $\mathrm{p}=0.38$, respectively) (Figure 1A). However, levels of TLR4 were elevated in PMN of nursing home residents (mean percent positive cells 33.91 [SE 2.75] vs. 15.67 [SE $1.58], \mathrm{p}<0.001)$.

As a measure of functional efficiency of PMNs, we assessed expression of $\beta 2$ integrins, key surface adhesion molecules that are up-

\begin{tabular}{|c|c|c|}
\hline & $\begin{array}{c}\text { Nursing home } \\
\text { residents } \\
n=15\end{array}$ & $\begin{array}{c}\text { Community } \\
\text { dwellers } \\
n=43\end{array}$ \\
\hline \multicolumn{3}{|l|}{ Demographics } \\
\hline Age in years - median (range) & $83(68-91)$ & $74(65-87)^{*}$ \\
\hline Gender - Female, n (\%) & $7(47)$ & $22(51)$ \\
\hline Race - White, n (\%) & $15(100)$ & $40(93)$ \\
\hline Ethnicity - Hispanic, n (\%) & $1(7)$ & NR \\
\hline Dementia - n (\%) & $7(47)$ & NR \\
\hline Number of co-morbidities - median (range) & $2(0-4)$ & $1(1-2)$ \\
\hline Number of medications - median (range) & $8(3-16)$ & NR \\
\hline \multicolumn{3}{|l|}{ Mental Status - Level of consciousness } \\
\hline Alert - n (\%) & $14(93)$ & NR \\
\hline \multicolumn{3}{|l|}{ ADLs in past 7 days } \\
\hline Bed mobility - dependent, n (\%) & $0(0)$ & NR \\
\hline Transfer - dependent, n (\%) & $1(7)$ & NR \\
\hline Locomotion off unit - dependent, n (\%) & $8(53)$ & NR \\
\hline Dressing - dependent, n (\%) & $4(27)$ & NR \\
\hline Eating - dependent, n (\%) & $0(0)$ & NR \\
\hline Toilet use - dependent, n (\%) & $3(20)$ & NR \\
\hline Personal hygiene - dependent, n (\%) & $3(20)$ & NR \\
\hline Bathing - dependent, n (\%) & $7(47)$ & NR \\
\hline Bowel incontinence, $\mathrm{n}(\%)$ & $1(7)$ & NR \\
\hline Bladder incontinence, $\mathrm{n}(\%)$ & $3(20)$ & NR \\
\hline
\end{tabular}

Table 1: Characteristics of nursing home residents and community dwellers. 


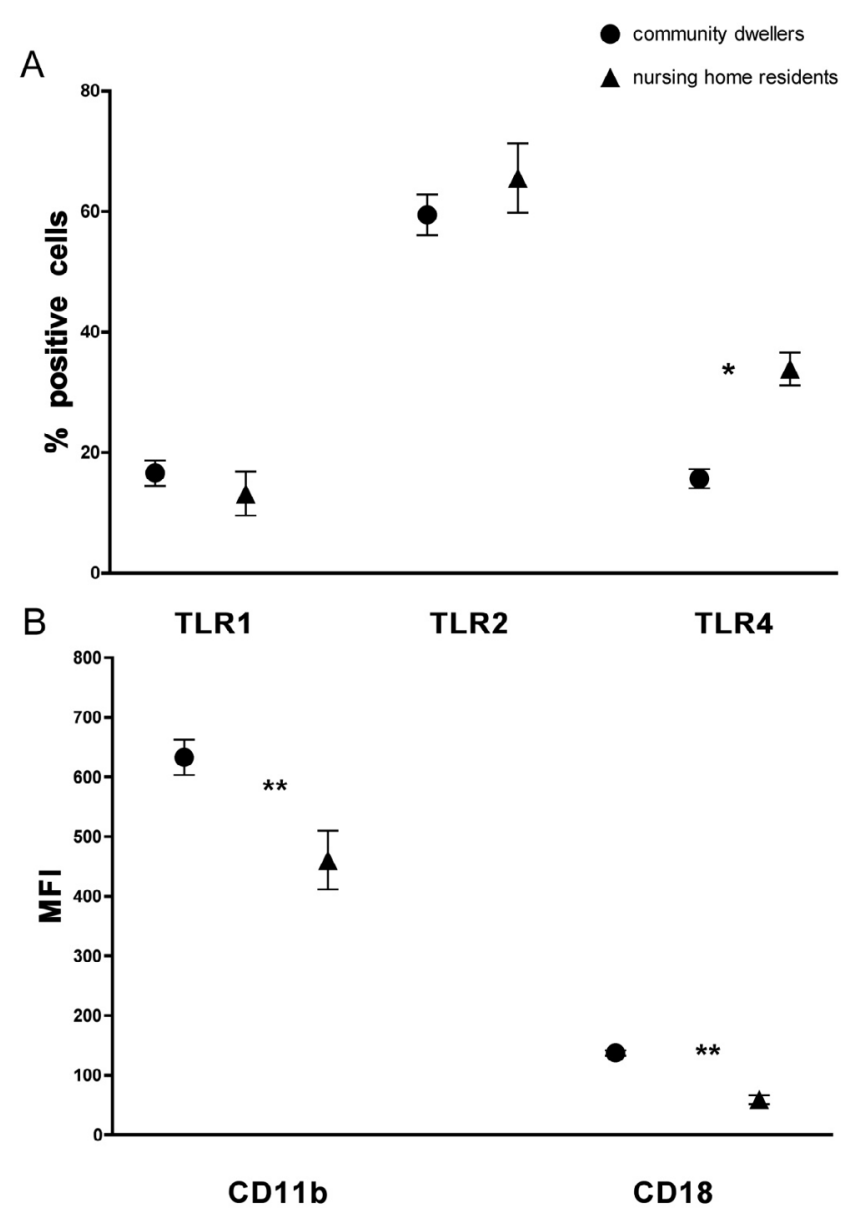

Figure 1: Altered Expression of PMN Markers in Nursing Home Elders Surface expression on PMN of healthy elderly $(n=41-44)$ and nursing home residents $(n=15)$ was quantified by FACS. Data shown are the mean values and $95 \%$ confidence intervals of the positive percentage of PMNs labeled for TLRs 1, 2, 4 (A) and the mean fluorescent intensity (MFI) for (B) $\square 2$ integrins CD11b and CD18. ${ }^{*} p<0.001$

${ }^{* *} p<0.0001$.

regulated on cell activation. ${ }^{10,11}$ When we compared levels of integrins, we noted dramatic reductions in both CD11b and CD18 (mean fluorescent intensity 460.8 [SE 49.1] vs. 632.9 [SE 29.5] and 59.6 [SE 7.9] vs. 137.6 [SE 4.6] respectively, $\mathrm{p}<0.0001$ ) from PMN of nursing home residents compared to PMN from healthy community-dwelling elders (Figure 1B).

\section{Lower induction of chemokines from PMN of nursing home residents}

To assess the efficiency of immune responsiveness in PMN of nursing home residents in comparison to community dwelling elders, we used qPCR to quantify levels of chemokines and cytokines produced by PMN. There were substantially reduced levels of the chemokines CCL4, CCL20, and $I L-8$ both at baseline and after stimulation by the TLR ligands LPS (TLR4) and Pam3CSK4 (TLR1/2), or by TNF- $a$, a key regulator of PMN activation (Figure 2A-2C).These PMN products are key for recruitment and activation of additional innate immune cells and lead to amplification of the immune response, thus any alterations will have important consequences for overall host responses.

\section{Discussion}

In this pilot study comparing the innate immune responses of neutrophils from nursing home residents versus community dwelling elders, we found that expression of the $\beta$-integrins CD11b and CD18 and chemokine induction were lower from neutrophils of nursing home residents. Expression of TLR1 and 2 did not significantly differ between the two groups, but expression of TLR4 was increased in PMNs from nursing home residents, compared to community-dwelling older adults. Since integrins are an important pathway to phagocyte
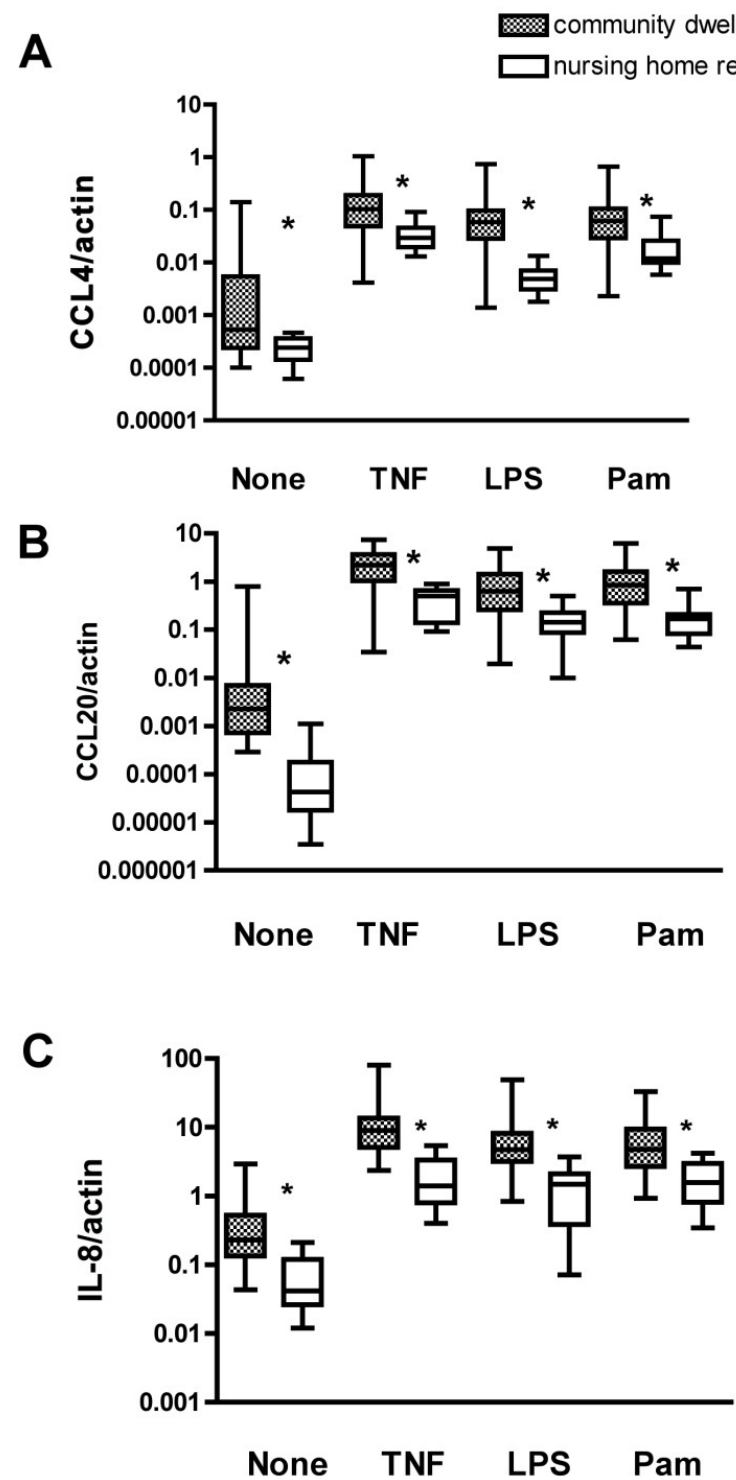

Figure 2: Reduced Production of Chemokines by PMN of Nursing Home Elders

Purified PMN of community dwellers $(n=41)$ and nursing home residents $(n=14)$ were assessed either untreated or following stimulation with TNF (20 $\mathrm{ng} / \mathrm{ml})$, Pam3CSK4 $(5 \mu \mathrm{g} / \mathrm{ml})$, or LPS $(0.5 \mu \mathrm{g} \mu \mathrm{g} / \mathrm{ml})$ for $30 \mathrm{~min}$ at $37^{\circ} \mathrm{C}$. mRNA levels of indicated chemokines were quantified by Q-PCR and normalized to $\beta$-actin. The median values and inter-quartile range are indicated for $(A) C C L 4$, (B) CCL20, and (C) IL-8. Statistical significance between community dwellers and nursing home residents by Mann Whitney, ${ }^{*} p<0.01$ 
Citation: Juthani-Mehta M, Guo X, Shaw AC, Towle V, Ning Y, et al. (2014) Innate Immune Responses in the Neutrophils of Community Dwelling and Nursing Home Elders. Aging Sci 2: 115. doi: 10.4172/2329-8847.1000115

Page 4 of 4

signaling and contribute to adherence and locomotion of neutrophils, [15] reduced $\beta 2$ integrin expression suggests that PMN from nursing home residents may have reduced responses to stimulation and reduced adhesive properties. Our observation of decreased CCL4, CCL20, and IL-8 gene expression at baseline and following TNF- $\alpha$, TLR4, and TLR1/2 stimulation is consistent with this hypothesis. The finding of increased surface TLR4 protein expression on PMNs from nursing home residents compared to community-dwelling older adults would appear to contrast with these results; however, recent data indicate that integrin CD11b negatively regulates TLR4 response, [15] suggesting that the lower levels of CD11b we observed could contribute to elevated expression of TLR4. The fact that TLR4-induced chemokine production is reduced in PMNs from nursing home residents despite increased TLR4 expression suggests an associated impairment in TLR4-dependent signal transduction in PMNs; additional studies are needed to address this question.

We were able to enroll only 15 nursing home residents into this study, and this reflects the difficulty in working with this vulnerable population. Large scale clinical trials are required to determine whether these findings are generalizable to the entire nursing home population. Nevertheless, these initial findings provide compelling data that can be further explored and validated in future cohorts.

Immunosenescence, in part, is thought to predispose older nursing home residents in particular to bacterial infections [5]. Elevated TLR4 expression, reduced $\beta 2$ integrin expression, and reduced chemokine induction among neutrophils all are examples of this dysregulation and may cause older nursing home residents to be at increased susceptibility to common bacterial infections such as pneumonia and UTI. Previous studies in monocytes and dendritic cells have identified reduced immune responses among community dwelling elders as compared to younger adults $[6,7]$. The findings from this study are the first analyses of neutrophil function in older nursing home residents, and demonstrate that even among older adults, these oldest institutionalized nursing home residents had more severely impaired innate immune responses compared to community-dwelling elders. Future studies should address possible mechanisms and implications of these observations to improve therapeutic strategies for institutionalized disabled elderly populations.

\section{Acknowledgements}

Conflict of Interest: No authors had any financial or personal conflicts

Author contributions: Study concept and design: MJM, AS, EF, RM; Acquisition of subjects and/or data: MJM, XG, AS, VT, XW, RM; Analysis and interpretation of data: MJM, AS, YN, HA, EF, RM; and Preparation of manuscript: MJM, XG, AS, VT, YN, XW, HA, EF, RM.

Sponsor's role: The sponsors had no role in the conduct of this study.

\section{Funding Sources}

This work was supported in part by the NIH (N01-HHSN272201100019C, NCRR/GCRC Program M01-RR00125, NIA K23 AG028691, Claude D. Pepper Older Americans Independence Center [P30 AG21342], and CTSA Grant Number UL1 RR024139 from the National Center for Research Resources [NCRR]). E.F. is an Investigator of the Howard Hughes Medical Institute. MJM is supported by Atlantic Philanthropies, IDSA/NFID, John A. Hartford Foundation, and Association of Specialty Professors.

\section{References}

1. High KP, Bradley SF, Gravenstein S, Mehr DR, Quagliarello VJ, et al. (2009) Clinical practice guideline for the evaluation of fever and infection in older adult residents of long-term care facilities: 2008 update by the Infectious Diseases Society of America. Clin Infect Dis. 48: 149-171.

2. High K (2007) Immunizations in older adults. Clin Geriatr Med 23: 669-685, viii-ix.

3. Jaillon S, Galdiero MR, Del Prete D, Cassatella MA, Garlanda C, et al. (2013) Neutrophils in innate and adaptive immunity. Semin Immunopathol 35: 377 394

4. Solana R, Tarazona R, Gayoso I, Lesur O, Dupuis G, et al. (2012) Innate immunosenescence: effect of aging on cells and receptors of the innate immune system in humans. Semin Immunol 24: 331-341.

5. Shaw AC, Goldstein DR, Montgomery RR (2013) Age-dependent dysregulation of innate immunity. Nat Rev Immunol 13: 875-887.

6. van Duin D, Mohanty S, Thomas V, Ginter S, Montgomery RR, et al. (2007) Age-associated defect in human TLR-1/2 function. J Immunol 178: 970-975.

7. Panda A, Qian F, Mohanty S, van Duin D, Newman FK, et al. (2010) Ageassociated decrease in TLR function in primary human dendritic cells predicts influenza vaccine response. J Immunol 184: 2518-2527.

8. Qian F, Wang X, Zhang L, Chen S, Piecychna M, et al. (2012) Age-associated elevation in TLR5 leads to increased inflammatory responses in the elderly. Aging Cell 11: 104-110.

9. Kong KF, Delroux K, Wang X, Qian F, Arjona A, et al. (2008) Dysregulation of TLR3 impairs the innate immune response to West Nile virus in the elderly. $J$ Virol 82: 7613-7623.

10. Kishimoto TK, Baldwin ET, Anderson DC (1999) The Role of ?2 Integrins in Inflammation. In: Gallin JI, Snyderman R, eds. Inflammation: Basic Principals and Clinical Correlates. 3rd ed. Phila: Lippincott, Williams \& Wilkins 537-569.

11. Schymeinsky J, Mócsai A, Walzog B (2007) Neutrophil activation via beta2 integrins (CD11/CD18): molecular mechanisms and clinical implications. Thromb Haemost 98: 262-273.

12. Juthani Mehta M, Quagliarello V, Perrelli E, Towle V, Van Ness PH, et al. (2009) Clinical features to identify urinary tract infection in nursing home residents: a cohort study. J Am Geriatr Soc 57: 963-970.

13. Hawes C, Morris JN, Phillips CD, Mor V, Fries BE, et al. (1995) Reliability estimates for the Minimum Data Set for nursing home resident assessment and care screening (MDS). Gerontologist 35: 172-178.

14. Guo X, Booth CJ, Paley MA, Wang X, DePonte K, et al. (2009) Inhibition of neutrophil function by two tick salivary proteins. Infect Immun 77: 2320-2329.

15. Han C, Jin J, Xu S, Liu H, Li N, et al. (2010) Integrin CD11b negatively regulates TLR-triggered inflammatory responses by activating Syk and promoting degradation of MyD88 and TRIF via Cbl-b. Nat Immunol 11: 734-742. 